\title{
Pattern Detection Using a Maximal Rejection Classifier
}

\author{
Michael Elad*, Yacov Hel-Or†, Renato Keshet \\ Hewlett-Packard Laboratories - Israel
}

\begin{abstract}
In this paper we propose a new classifier - the Maximal Rejection Classifier (MRC) - for target detection. Unlike pattern recognition, pattern detection problems require a separation between two classes, Target and Clutter, where the probability of the former is substantially smaller, compared to that of the latter. The MRC is a linear classifier, based on successive rejection operations. Each rejection is performed using a projection followed by thresholding. In contrast to common classifiers the projection vector is influenced by the probabilities of obtaining target or clutter signals. The projection vector is designed to minimize the expected number of operations until detection. In the case where the probabilities of target and clutter signals are equal, it is shown that the Fisher linear discriminant is optimal in the above sense. However, in more common cases where the probablitities are quite different, a new optimal classifier is suggested. An application of detecting frontal faces in images is demonstrated using the MRC with encouraging results.
\end{abstract}

${ }^{*}$ Currently with Jigami Corporation, The Technion City, Haifa 32000 Israel, email: elad@jigami.com

${ }^{\dagger}$ Currently at the Interdisciplinary Center (IDC), Herzlia, Israel, email: toky@idc.ac.il 


\section{Introduction}

In target detection applications, the aim is to detect occurrences of a specific Target in a given signal. In general, the target is subjected to some particular type of transformation, hence we have a set of target signals to be detected. In this context, the set of non-Target samples are referred to as Clutter. In practice, the target detection problem can be characterized as designing a classifier $C(z)$, which, given an input vector $z$, has to decide whether $z$ belongs to the Target class $\mathbf{X}$ or the Clutter class $\mathbf{Y}$. In example based classification, this classifier is designed using two training sets $-\hat{\mathbf{X}}=\left\{x_{i}\right\}_{i=1 . . L_{x}}$ (Target samples) and $\hat{\mathbf{Y}}=\left\{y_{i}\right\}_{i=1 . . L_{y}}$ (Clutter samples), drawn from the above two classes.

Since the classifier $C(z)$ is usually the heart of a detection algorithm, and is applied many times, simplifying it translates immediately to an efficient detection algorithm. Various types of example-based classifiers are suggested in the literature $[1,2,3]$. The most simple and fast are the linear classifiers, where $C(z)$ is based on a projection operation followed by a thresholding. The projection of $z$ is performed onto a projection vector $u$, thus, $C(z)=f\left(u^{t} z\right)$ where $f(*)$ is a thresholding operation (or some other decision rule). The Support Vector Machine (SVM) [2] and the Fisher Linear Discriminant (FLD) [1] are two examples of linear classifiers. In both cases the kernel $u$ is chosen in some optimal manner. In the FLD, $u$ is chosen such that the Mahalanobis distance of the two classes after projection will be maximized. In the SVM approach the motive is similar, but the vector $u$ is chosen such that it maximizes the margin between the two sets.

In both these classifiers, it is assumed that the two classes have equal importance. In typical target detection applications the above assumption is not valid since the probability of $z$ belonging to $\mathbf{X}$ is substantially smaller, compared to that of belonging to $\mathbf{Y}$. Both the FLD and the SVM do not exploit this property. Moreover, in both of these methods, it is assumed that the classes 
are linearly separable. in order to be able to treat more complex, and unfortunately, more common scenarios, non-linear extensions of these algorithms are required $[1,2]$. Such extensions are typically at the expense of much more computationally intensive algorithms.

The Maximal Rejection Classifier (MRC) is a linear classifier that overcomes the above two drawbacks. While maintaining the simplicity of a linear classifier, it can also deal with non linearly separable cases. The only requirement is that the Clutter class and the convex hull of the Target class are disjoint. We define this property as two convexly-separable classes, which is a much weaker condition compared to linear-separability. In addition, this classifier exploits the property of high Clutter probability. Hence, it attempts to give very fast Clutter labeling, even if at the expense of slow Target labeling. Thus, the entire input signal is classified very fast.

The MRC is an iterative rejection based classification algorithm. The main idea is to apply at each iteration a linear projection followed by a thresholding, similar to the SVM and the FLD. However, as opposed to these two methods, the projection vector and the corresponding thresholds are chosen such that at each iteration the MRC attempts to maximize the number of rejected Clutter samples. This means that following the first classification iteration, many of the Clutter samples are already classified as such, and discarded from further consideration. The process is continued with the remaining Clutter samples, again searching for a linear projection vector and thresholds that maximizes the rejection of Clutter points from the remaining set. This process is repeated iteratively until a small number or non of the Clutter points remain. The remaining samples at the final stage are considered as Targets. The idea of rejection-based classifier was already introduced by [3]. However, in this work we extend the idea by using maximal rejection.

In order to demonstrate the behavior of the MRC, this algorithm is applied to the problem of 
detecting frontal and vertical faces in images. It is demonstrated that the MRC is a very efficient algorithm, requiring an effective computation of close to two convolutions of the input image per each resolution layer in order to reliably detect faces at all scales and all spatial positions.

\section{The MRC in Theory}

Assume two classes are given in $\Re^{n}, \mathbf{X}$ (the Target class) and $\mathbf{Y}$ (the Clutter class). It is required to discriminate between these two classes, i.e., given a point $z$ drawn from one of these classes, we would like to be able to label it correctly as either Target or Clutter. One important point, however, is that we know a-priori that for a typical input stream the vast majority of the inputs are Clutters, i.e.:

$$
P\{\mathbf{X}\} \ll P\{\mathbf{Y}\}
$$

where $P\{\mathbf{X}\}$ is the a-priori probability that an input signal will be a Target, and $P\{\mathbf{Y}\}$ is defined similarly. Based on this knowledge, we would like the classifier to give a decision as fast as possible (i.e., with as few operations as possible). Thus, Clutter labeling should be performed fast, even if at the expense of slow Target labeling.

Similar to other linear classifiers $[1,2]$, we suggest to first project the sample $z$ onto a vector $u$, and label it based on the projected value $\alpha=u^{T} z$. Projecting the Target class onto $u$ results with a Probability Density Function (PDF) $P\{\alpha \mid \mathbf{X}\}$, defined as:

$$
P\{\alpha \mid \mathbf{X}\}=\int_{z} P\{z \mid \mathbf{X}\} \delta\left(u^{T} z-\alpha\right) d z
$$

where $\delta(x)$ is the Dirac's function. The term $P\{\alpha \mid \mathbf{X}\}$ defines the probability that a given input drawn from the Target class will obtain the value $\alpha$ after it has been projected onto $u$. Similarly, 
Projecting the Clutter class onto $u$ results with a $\operatorname{PDF} P\{\alpha \mid \mathbf{Y}\}$ :

$$
P\{\alpha \mid \mathbf{Y}\}=\int_{z} P\{z \mid \mathbf{Y}\} \delta\left(u^{T} z-\alpha\right) d z
$$

We define the following intervals based on $P\{\alpha \mid \mathbf{X}\}$ and $P\{\alpha \mid \mathbf{Y}\}$ :

$$
\begin{aligned}
C_{t} & =\{\alpha \mid P\{\alpha \mid \mathbf{X}\}>0, P\{\alpha \mid \mathbf{Y}\}=0\} \\
C_{c} & =\{\alpha \mid P\{\alpha \mid \mathbf{X}\}=0, P\{\alpha \mid \mathbf{Y}\}>0\} \\
C_{u} & =\{\alpha \mid P\{\alpha \mid \mathbf{X}\}>0, P\{\alpha \mid \mathbf{Y}\}>0\}
\end{aligned}
$$

( $t$-Target, $c$-Clutter and $u$-Unknown). After projection, $z$ is labeled either as a Target, Clutter, or Unknown, based on the following decision rule:

$$
\text { Classifier }\{z\}= \begin{cases}\text { Target } & u^{T} z \in C_{t} \\ \text { Clutter } & u^{T} z \in C_{c} \\ \text { Unknown } & u^{T} z \in C_{u}\end{cases}
$$

Unknown classifications are obtained only in the $C_{u}$ interval, where a decision cannot be made. Figure 1 presents an example for the construction of the intervals $C_{t}, C_{c}$ and $C_{u}$ and their appropriate decisions. The probability of the Unknown decision is given by:

$$
P\{\text { Unknown }\}=\int_{\alpha \in C_{u}} P\{\mathbf{Y}\} P\{\alpha \mid \mathbf{Y}\} d \alpha+\int_{\alpha \in C_{u}} P\{\mathbf{X}\} P\{\alpha \mid \mathbf{X}\} d \alpha
$$

The above term is a function of the projection vector $u$. We would like to find the vector $u$ which minimizes the "Unknown" probability. However, since this is a complex minimization problem, an alternative minimization is developed here, using a proximity measure between the two PDF's.

If $P\{\alpha \mid \mathbf{Y}\}$ and $P\{\alpha \mid \mathbf{X}\}$ are far apart and separated from each other $P\{$ Unknown $\}$ will be small. Therefore, an alternative requirement is to minimize the overlap between these two PDF's. We will define this requirement using the following expected distance between a point $\alpha_{0}$ and a distribution $P\{\alpha\}$ :

$$
D\left(\alpha_{0} \| P\{\alpha\}\right)=\int_{\alpha} \frac{\left(\alpha_{0}-\alpha\right)^{2} P\{\alpha\}}{\sigma^{2}} d \alpha
$$




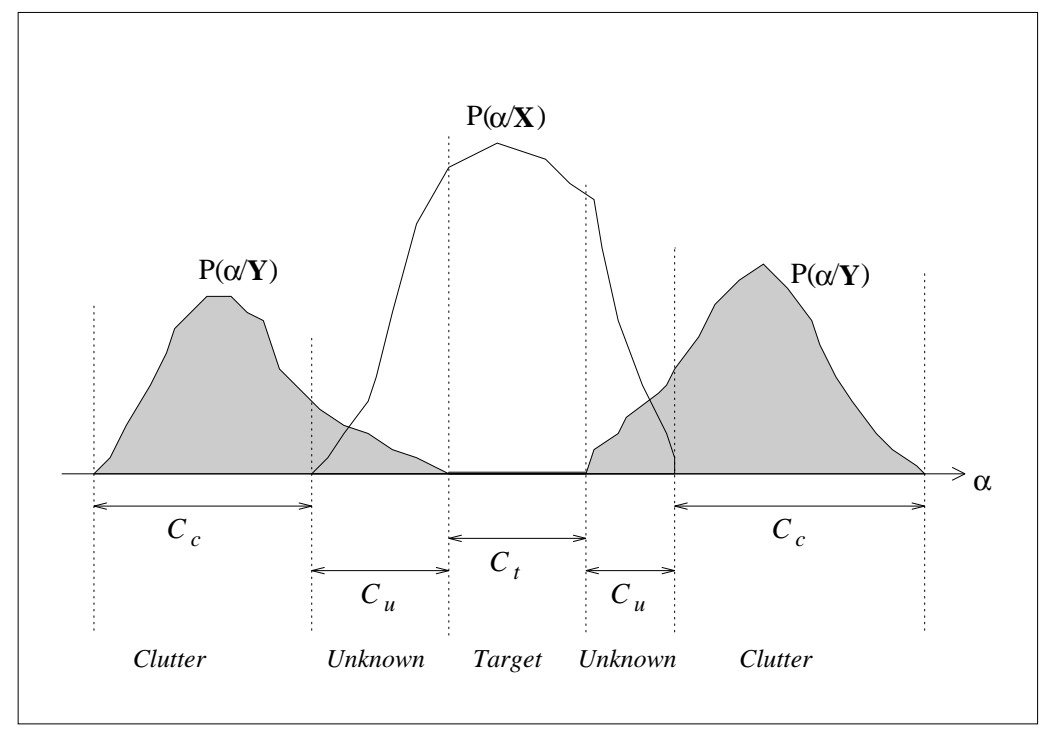

Figure 1: The intervals $C_{t}, C_{c}$ and $C_{u}$, for specific PDFs $P\{\alpha \mid \mathbf{X}\}$ and $P\{\alpha \mid \mathbf{Y}\}$.

where $\sigma$ is the variance of $P\{\alpha\}$ and the division by $\sigma$ is performed in order to make the distance scale-invariant (or unit-invariant). Calculating the integral above, it is easy to verify that:

$$
D\left(\alpha_{0} \| P\{\alpha\}\right)=\frac{\left(\alpha_{0}-\mu\right)^{2}+\sigma^{2}}{\sigma^{2}}
$$

where $\mu$ is the mean of $P\{\alpha\}$. Using this distance definition, the distance of $P\{\alpha \mid \mathbf{X}\}$ from $P\{\alpha \mid \mathbf{Y}\}$ can be defined as the expected distance of $(\alpha \mid \mathbf{Y})$ from $P\{\alpha \mid \mathbf{X}\}$ :

$$
\begin{aligned}
D(P\{\alpha \mid \mathbf{Y}\} \| P\{\alpha \mid \mathbf{X}\}) & =\int_{\alpha} D(\alpha \| P\{\alpha \mid \mathbf{X}\}) P\{\alpha \mid \mathbf{Y}\} d \alpha= \\
= & \int_{\alpha} \frac{\left(\alpha-\mu_{x}\right)^{2}+\sigma_{x}^{2}}{\sigma_{x}^{2}} P\{\alpha \mid \mathbf{Y}\} d \alpha=\frac{\left(\mu_{y}-\mu_{x}\right)^{2}+\sigma_{x}^{2}+\sigma_{y}^{2}}{\sigma_{x}^{2}}
\end{aligned}
$$

where $\left[\mu_{x}, \sigma_{x}\right]$ and $\left[\mu_{y}, \sigma_{y}\right]$ are the mean-variance pairs of $P\{\alpha \mid \mathbf{X}\}$ and $P\{\alpha \mid \mathbf{Y}\}$, respectively. Since we want the two distributions to have as small an overlap as possible, we would like to maximize this distance or minimize the proximity between $P\{\alpha \mid \mathbf{Y}\}$ and $P\{\alpha \mid \mathbf{X}\}$, which can be defined as the inverse of their mutual distance. Note, that this measure is asymmetric with respect to the two distributions, i.e the proximity defines the closeness of $P\{\alpha \mid \mathbf{Y}\}$ to $P\{\alpha \mid \mathbf{X}\}$, but not vice versa. Therefore, we define the overall proximity between the two distributions as 
follows:

$$
\operatorname{Prox}(P\{\alpha \mid \mathbf{Y}\}, P\{\alpha \mid \mathbf{X}\})=P\{\mathbf{X}\} \frac{\sigma_{y}^{2}}{\sigma_{x}^{2}+\sigma_{y}^{2}+\left(\mu_{y}-\mu_{x}\right)^{2}}+P\{\mathbf{Y}\} \frac{\sigma_{x}^{2}}{\sigma_{x}^{2}+\sigma_{y}^{2}+\left(\mu_{y}-\mu_{x}\right)^{2}}
$$

Compared to the original expression in Equation 6, the minimization of this term with respect to $u$ is easier. If $P\{\mathbf{X}\}=P\{\mathbf{Y}\}$, i.e. if there is an even chance to obtain Target or Clutter inputs, the proximity becomes:

$$
\operatorname{Prox}(P\{\alpha \mid \mathbf{Y}\}, P\{\alpha \mid \mathbf{X}\})=\frac{\sigma_{x}^{2}+\sigma_{y}^{2}}{\sigma_{x}^{2}+\sigma_{y}^{2}+\left(\mu_{y}-\mu_{x}\right)^{2}}
$$

which is the cost function minimized by the Fisher Linear Discriminant (FLD)[1]. In our case $P\{\mathbf{X}\} \ll P\{\mathbf{Y}\}$ (Equation 1), thus, the first term is negligible in Equation 9 and can be omitted. Therefore, the optimal $u$ should minimize the resulting term:

$$
d(u)=\frac{\sigma_{x}^{2}}{\sigma_{x}^{2}+\sigma_{y}^{2}+\left(\mu_{y}-\mu_{x}\right)^{2}}
$$

where $\sigma_{y}^{2}, \sigma_{x}^{2}, \mu_{y}$ and $\mu_{x}$ are all a function of the projection vector $u$.

Minimization of this expression usually results in a small $\sigma_{x}$ and large $\sigma_{y}$. This means that the projection of Target inputs tend to concentrate near a constant value, whereas the Clutter inputs will spread with a large variance (see e.g. Fig 2) .

For the optimal $u$, most of the Clutter inputs will be projected onto $C_{c}$, while $C_{t}$ might even be an empty set. Subsequently, after projection, many of the Clutter inputs are usually classified, whereas Target labeling may not be immediately possible. This serves our purpose because for a Clutter input, there is a high probability that a decision will be made. Since these inputs are more frequent, this means a faster decision for the vast majority of the inputs.

The method which we suggest follows this scheme: The classifier works in an iterative manner, projecting and thresholding with different parameters at each iteration sequentially. Since the 
classifier is asymmetric, the classification is based on rejections; Clutter inputs are classified and removed from further consideration while the remaining inputs are kept as suspected Targets. The iterations and the rejection approaches are both key concepts of the proposed scheme.

\section{The MRC in Practice}

Let us return to Equation 11 and find the optimal projection vector $u$. In order to do so, we have to express $\sigma_{y}^{2}, \sigma_{x}^{2}, \mu_{y}$ and $\mu_{x}$ as functions of $u$. It is easy to see that:

$$
\mu_{x}=u^{T} \mathbf{M}_{x} \quad \text { and } \quad \sigma_{x}^{2}=u^{T} \mathbf{R}_{x x} u
$$

and similarly

$$
\mu_{y}=u^{T} \mathbf{M}_{y} \quad \text { and } \quad \sigma_{y}^{2}=u^{T} \mathbf{R}_{y y} u
$$

where we define:

$$
\begin{array}{ll}
\mathbf{M}_{x}=\int_{z} z P\{z \mid \mathbf{X}\} d z & \mathbf{R}_{x x}=\int_{z}\left(z-\mathbf{M}_{x}\right)\left(z-\mathbf{M}_{x}\right)^{T} P\{z \mid \mathbf{X}\} d z \\
\mathbf{M}_{y}=\int_{z} z P\{z \mid \mathbf{Y}\} d z & \mathbf{R}_{y y}=\int_{z}\left(z-\mathbf{M}_{y}\right)\left(z-\mathbf{M}_{y}\right)^{T} P\{z \mid \mathbf{Y}\} d z .
\end{array}
$$

As can be seen, only the first and second moments of the classes play a role in the choice of the projection vector $u$.

In practice we usually do not have the the probabilities $P\{z \mid \mathbf{X}\}, P\{z \mid \mathbf{Y}\}$, and inference on the Target or Clutter class is achieved through examples. For the two example-sets $\hat{\mathbf{X}}=\left\{x_{k}\right\}_{k=1}^{L_{x}}$ and $\hat{\mathbf{Y}}=\left\{y_{k}\right\}_{k=1}^{L_{y}}$, the mean-covariance pairs $\left(\mathbf{M}_{x}, \mathbf{R}_{x x}, \mathbf{M}_{y}\right.$, and $\left.\mathbf{R}_{y y}\right)$ are replaced with empirical approximations:

$$
\begin{array}{ll}
\hat{\mathbf{M}}_{x}=\frac{1}{L_{x}} \sum_{k=1}^{L_{x}} x_{k} & \hat{\mathbf{R}}_{x x}=\frac{1}{L_{x}} \sum_{k=1}^{L_{x}}\left(x_{k}-\hat{\mathbf{M}}_{x}\right)\left(x_{k}-\hat{\mathbf{M}}_{x}\right)^{T} \\
\hat{\mathbf{M}}_{y}=\frac{1}{L_{y}} \sum_{k=1}^{L_{y}} y_{k} & \hat{\mathbf{R}}_{y y}=\frac{1}{L_{y}} \sum_{k=1}^{L_{y}}\left(y_{k}-\hat{\mathbf{M}}_{y}\right)\left(y_{k}-\hat{\mathbf{M}}_{y}\right)^{T}
\end{array}
$$


The function we aim to minimize is therefore:

$$
d(u)=\frac{u^{T} \hat{\mathbf{R}}_{x x} u}{u^{T}\left[\hat{\mathbf{R}}_{x x}+\hat{\mathbf{R}}_{y y}+\left(\hat{\mathbf{M}}_{y}-\hat{\mathbf{M}}_{x}\right)\left(\hat{\mathbf{M}}_{y}-\hat{\mathbf{M}}_{x}\right)^{T}\right] u}
$$

Similarly to $[1,4,5]$, it is easy to show that $u$ that minimizes the above expression satisfies:

$$
\hat{\mathbf{R}}_{x x} u=\lambda\left[\hat{\mathbf{R}}_{x x}+\hat{\mathbf{R}}_{y y}+\left(\hat{\mathbf{M}}_{y}-\hat{\mathbf{M}}_{x}\right)\left(\hat{\mathbf{M}}_{y}-\hat{\mathbf{M}}_{x}\right)^{T}\right] u
$$

and should correspond to the smallest possible $\lambda$. A problem of the form $A u=\lambda B u$, as in Equation 17, is known as the generalized eigenvalue problem [1, 4, 5], and has a closed form solution. Notice that given any solution $u$ for this equation, $\beta u$ is also a solution with the same $\lambda$. Therefore, without loss of generality, the normalized solution $\|u\|=1$ is used.

After finding the optimal projection vector $u$, the intervals $C_{t}, C_{c}$, and $C_{u}$ can be determined according to Equation 4. An input $z$ is labeled as a Target or Clutter if its projected value $u^{T} z$ is in $C_{t}$ or $C_{c}$, respectively. Figure 2 presents this stage for the case where $C_{t}$ is empty, i.e. there are no inputs which can be classified as Target.

Input vectors whose projected values are in $C_{u}$ are not labeled. For these inputs we apply another step of classification, where the design of the optimal projection vector in this step is performed according to the following new distributions:

$$
P\left\{z \mid \mathbf{Y} \& u_{1}^{T} z \in C_{u}\right\} \quad \text { and } \quad P\left\{z \mid \mathbf{X} \& u_{1}^{T} z \in C_{u}\right\}
$$

We define the next projection vector $u_{2}$ as the vector which minimizes the following proximity measure:

$$
\operatorname{Prox}\left(P\left\{u_{2}^{T} z \mid \mathbf{Y} \& u_{1}^{T} z \in C_{u}\right\}, P\left\{u_{2}^{T} z \mid \mathbf{X} \& u_{1}^{T} z \in C_{u}\right\}\right)
$$

This minimization is performed in the same manner as described for the first step. Figure 3 presents the second rejection stage, which follows the first stage shown in Figure 2. 


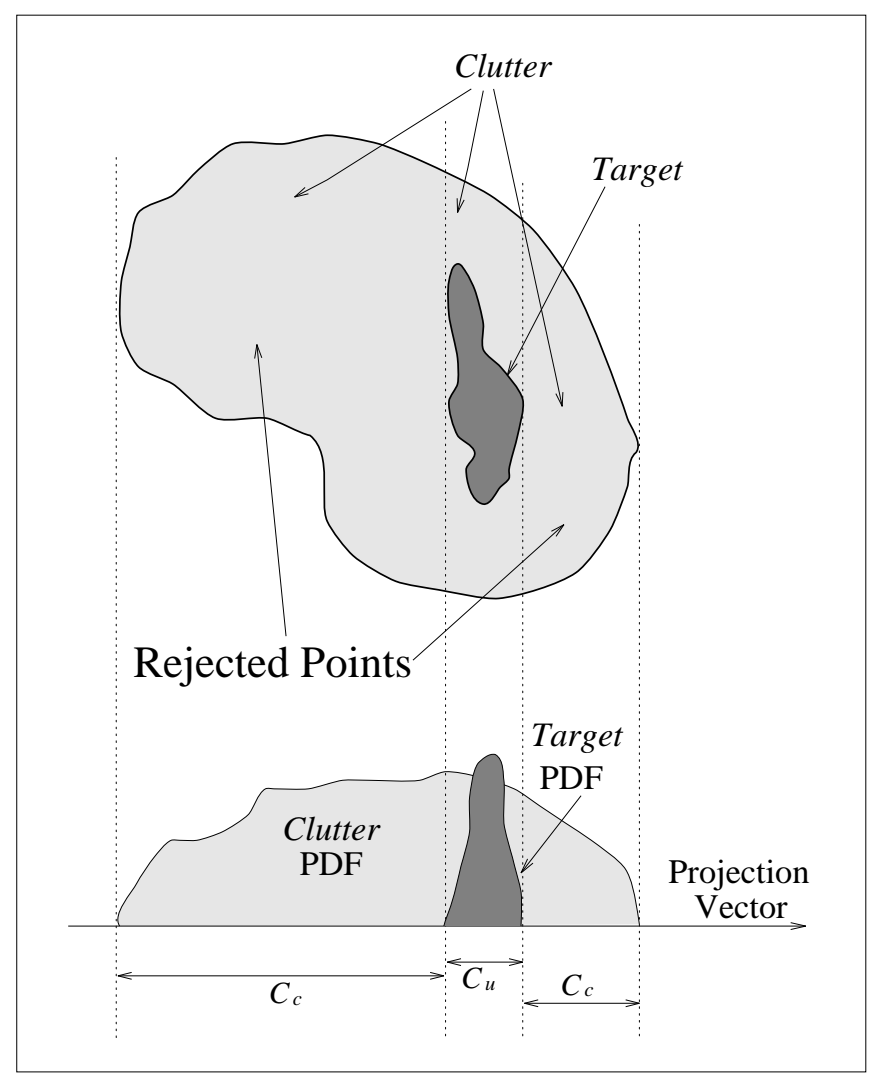

Figure 2: The first rejection stage for a 2D example.

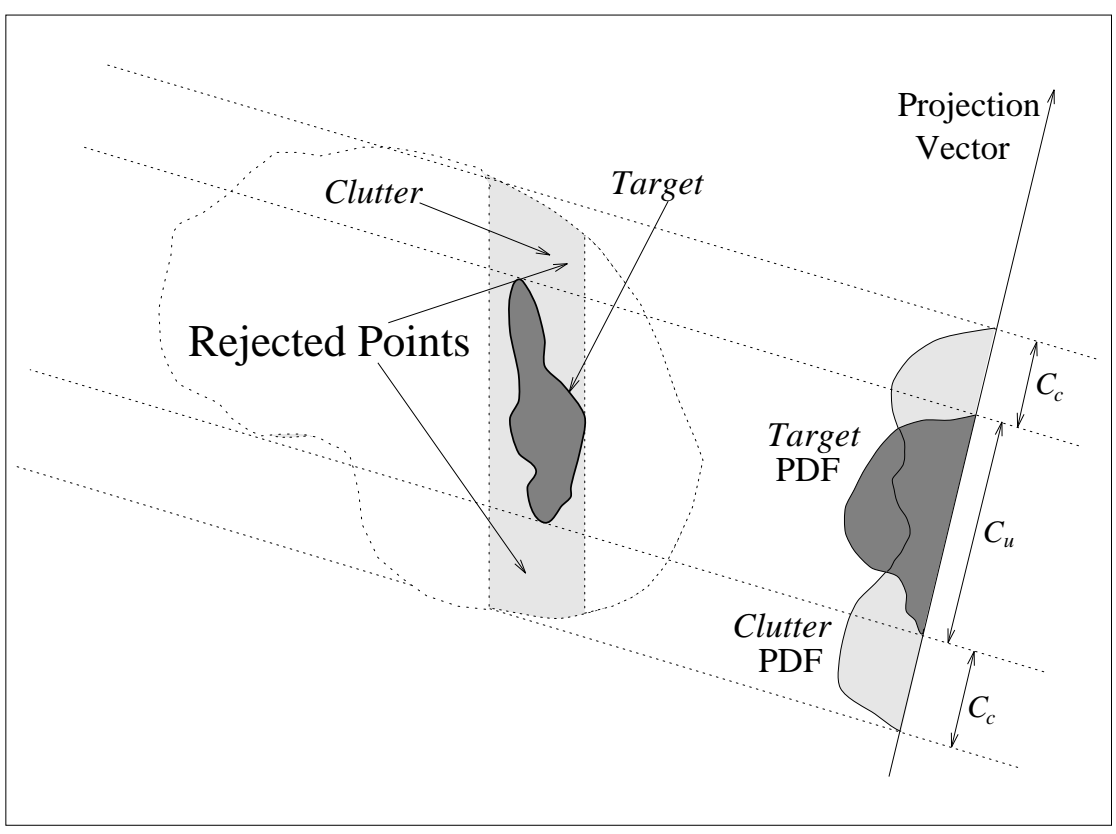

Figure 3: The second rejection stage. 
Following the second step, the process continues similarly with projection vectors $u_{3}, u_{4}, \cdots$, etc. Due to the optimality of the projection vector at each step, it is expected that a large portion of the input vectors will be labeled as Clutter at each step, while following steps will deal with the remaining input vectors. Applying the cascade of classifiers in such an iterative manner ensures a good performance of the classification with respect to an accurate labeling and a fast classification rate.

Since we exchanged the class probabilities with sets of points, it is impractical to define the intervals $C_{t}, C_{c}$, and $C_{u}$ using Equation 4. This is because the intervals will be composed of many fragments each of which results from a particular example. Moreover, the domain of $\alpha$ cannot be covered by a finite set of examples. Therefore, it is more natural to define for each set, two thresholds bounding its projection values. As explained above, due to the functional that we are minimizing, in most cases the Target thresholds define a small interval located inside the Clutter interval (see Figure 2). Therefore for simplicity, we define only a single interval $\Gamma=\left[T_{1}, T_{2}\right]$, which is the interval bounding the Target set, where we classify points projected outside $\Gamma$ as Clutter and points projected inside $\Gamma$ as Unknown.

In the case where the Target class forms a convex set, and the two classes are disjoint, it is theoretically possible to completely discriminate between them. More generally, if there are no Clutter inputs inside the convex hull of the Target set, exact discrimination is theoretically possible. This property is easily shown by noticing that we are actually extracting the Target set from the Clutter set by a sequence of two parallel hyper-planes, corresponding to the two thresholding operations. This constructs a parallelogram (see Figure 4 (a)) that bounds the Target set from outside. Since any convex set can be constructed by a set of parallel hyperplanes, exact classification is possible. However, if the Target set is non-convex, or the two classes are non-convexly separable (as defined in the Introduction), it is impossible to achieve 
a classification with zero errors; Clutters inputs which are inside the convex hull of the Target set cannot be rejected. Figure 4 (b) presents such a case. Overcoming this limitation can be accomplished by a non-linear extension of the MRC, which is outside the scope of this paper.

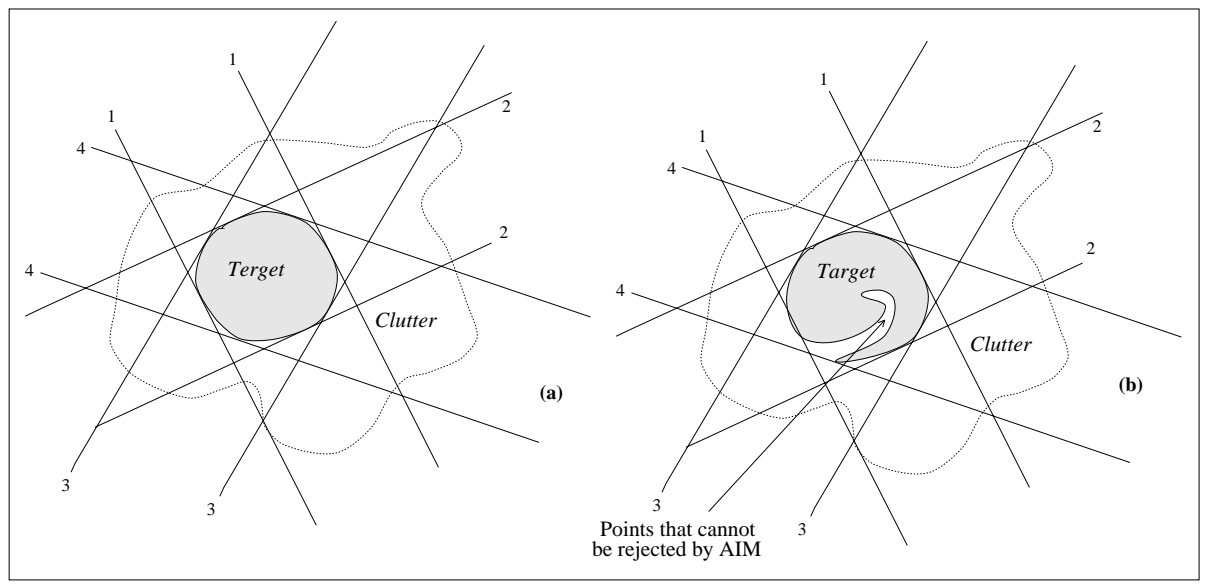

Figure 4: (a) Rejection of Clutter inputs for a convex Target set. (b) Rejection of Clutter inputs for a non-convex Target set.

In practice, even if we deal with a convex Target set, false-alarms may exist due to the the sub-optimal approach we are using, which neglects multi-dimensional moments higher than the second. However, simulations demonstrate that the number of false-alarms is typically small.

\section{Face Detection Using the MRC}

The face detection problem can be specified as the need to detect all instances of faces in a given image, at all spatial positions, all scales, all facial expressions, all poses, of all people, and under all lighting conditions. All these requirements should be met, while having few or no false alarms and miss-detections, and with as fast an algorithm as possible. This description reveals the complexity of the detection problem at hand. As opposed to other pattern detection problems, faces are expected to appear with considerable variations, even for detecting only frontal and vertical faces. Variations are expected because of changes in skin color, facial hair, 
glasses, face shape, and more.

Several papers already addressed the face detection problem using various methods, such as SVM $[2,6]$, Neural Networks [7, 8, 9], and other methods [10, 11, 12, 13]. In all of these studies, the above complete list of requirements is relaxed in order to obtain practical detection algorithms. Following $[6,7,9,10,13]$, we deal with the detection of frontal and vertical faces only.

In all these algorithms, spatial position and scale are treated through the same method, in which the given image is decomposed into a Gaussian pyramid with near-unity (e.g., 1.2) resolution ratio. The search for faces is performed in each resolution layer independently, thus enabling the treatment of different scales. In order to be able to detect faces at all spatial positions, fixed sized blocks of pixels are extracted from the image at all positions (with full or partial overlap) for testing. In addition to the pyramid part, which treats varying scales and spatial positions, the core part of the detection algorithm is essentially a classifier which provides a Face/Non-Face decision for each input block.

In this paper we propose the application of the MRC for this task. In the face-detection application, Faces take the role of targets, and Non-Faces are the clutter. In a typical image having millions of pixels, it is expected to detect a few dozens of faces at the most, which means that picking a Non-Face block from the image is much more probable. This property is exploited by the MRC in order to obtain an efficient face-detection classifier. The MRC produces very fast Non-Face labeling (i.e., with a low computational cost), at the expense of slow Face labeling. Thus, on the average, it has a short decision time per input block.

The first stage in the MRC is to gather two example-sets, Faces and Non-Faces. As mentioned earlier, large enough sets are needed in order to guarantee good generalization for the faces and 
the non-faces that may be encountered in images. As to the Face set, the ORL data-base ${ }^{1}$ was used. This database contains 400 frontal and vertical face images of 40 different individuals. By extracting the face portion from each of these images and scaling to $15 \times 15$ pixels, we obtained the set $\hat{\mathbf{X}}=\left\{x_{k}\right\}_{k=1}^{L_{x}}\left(\right.$ with $\left.L_{x}=400\right)$.

The Non-Face set is required to be much larger, in order to represent the variability of NonFace patterns in images. We took 54 arbitrary images containing various textures, natural scenes, graphic images, etc. Common to all these images is that they contain no faces. Each of the 54 images was decomposed into a Gaussian pyramid with a 1.2 resolution ratio, thus creating 1290 images. Using the pyramids is beneficial both for enriching the Non-Face set, and for including multi-resolution versions of the same patterns in the data-base. Each possible block of $15 \times 15$ pixels in these 1290 images is considered as a candidate example of Non-Face. Thus, we have effectively collected more than 20 million Non-Face examples.

\section{Results}

We trained the MRC for detecting faces by computing 50 sets of kernels $\left\{u_{k}\right\}_{k=1}^{50}$ and associated thresholds $\left\{\left[T_{1}^{k}, T_{2}^{k}\right]\right\}_{k=1}^{50}$, using the above described databases of Faces and Non-Faces. The following figures show the obtained results for several images.

In all these examples, the first stage rejected close to $90 \%$ of the candidates. This stage is merely a convolution of the input image (at every scale) with the first kernel, $u_{1}$, followed by thresholding. For these examples, the complete MRC classification required an effective number of close to two convolutions per each pixel in each resolution layer. As can be seen, there are few false alarms, which typically correspond to blocks of pixels having a pattern which may resemble

\footnotetext{
${ }^{1} \mathrm{http}$ ///www.cam-orl.co.uk/facedatabase.html: ORL database web-site
} 


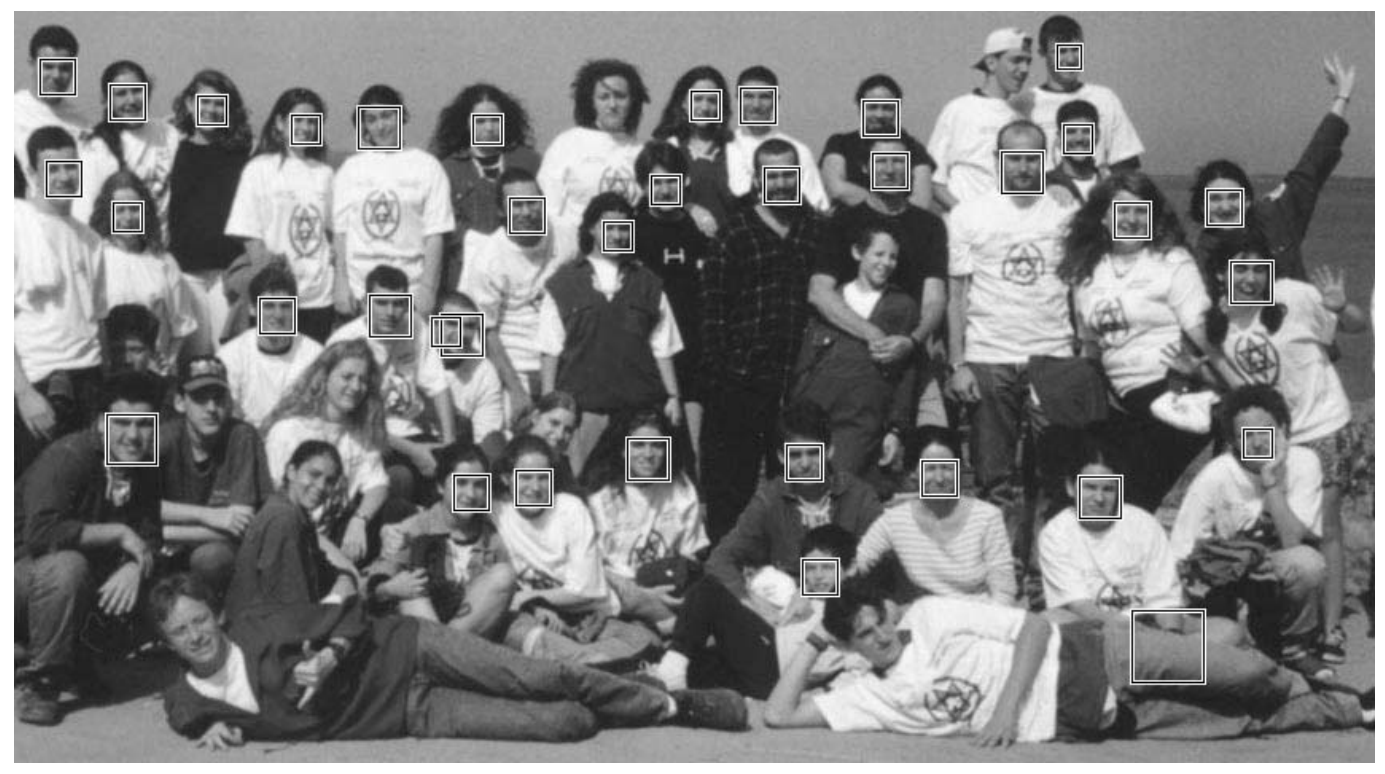

Figure 5: Face detection with the MRC - Example 1

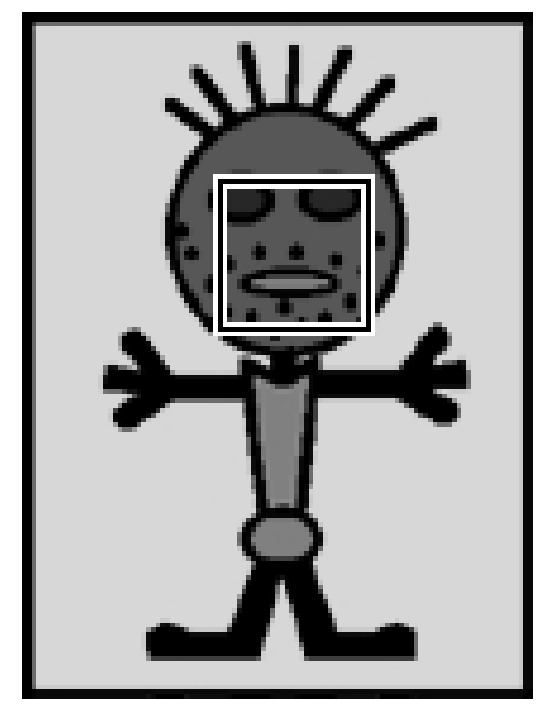

Figure 6: Face detection with the MRC - Example 2 


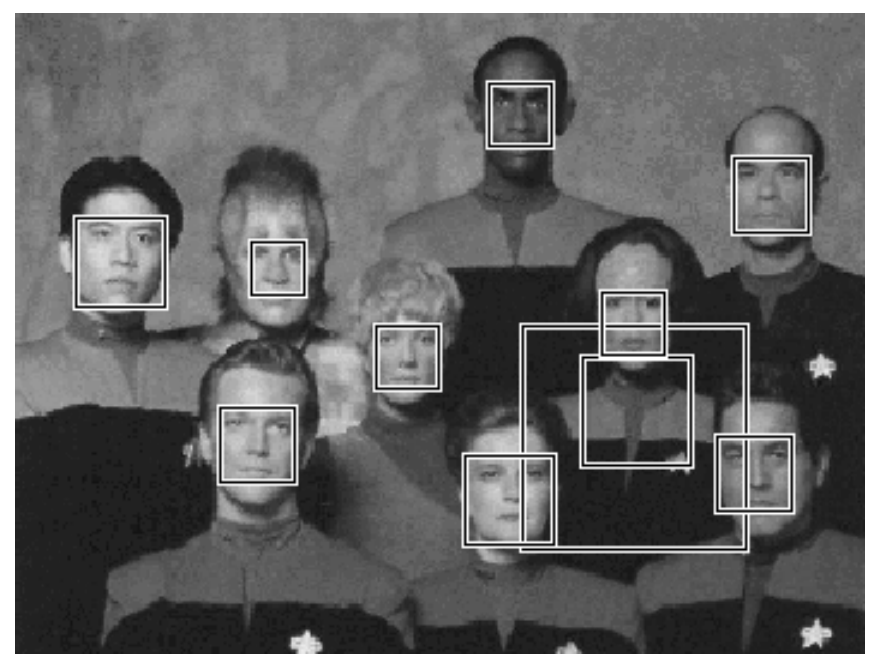

Figure 7: Face detection with the MRC - Example 3

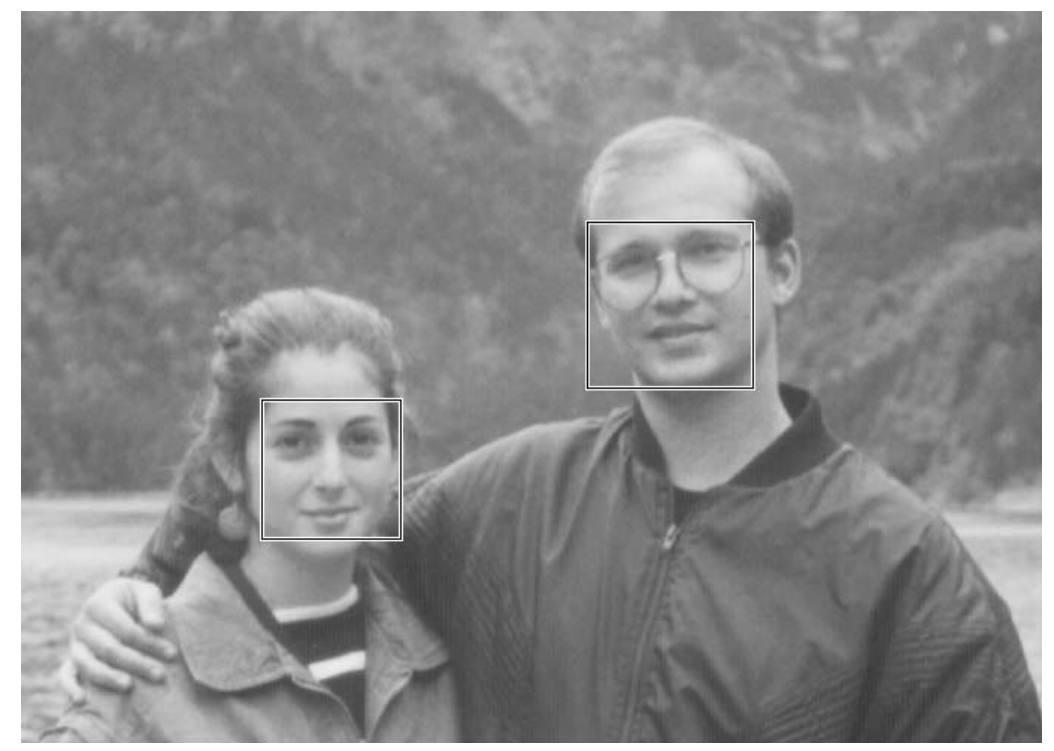

Figure 8: Face detection with the MRC - Example 4 


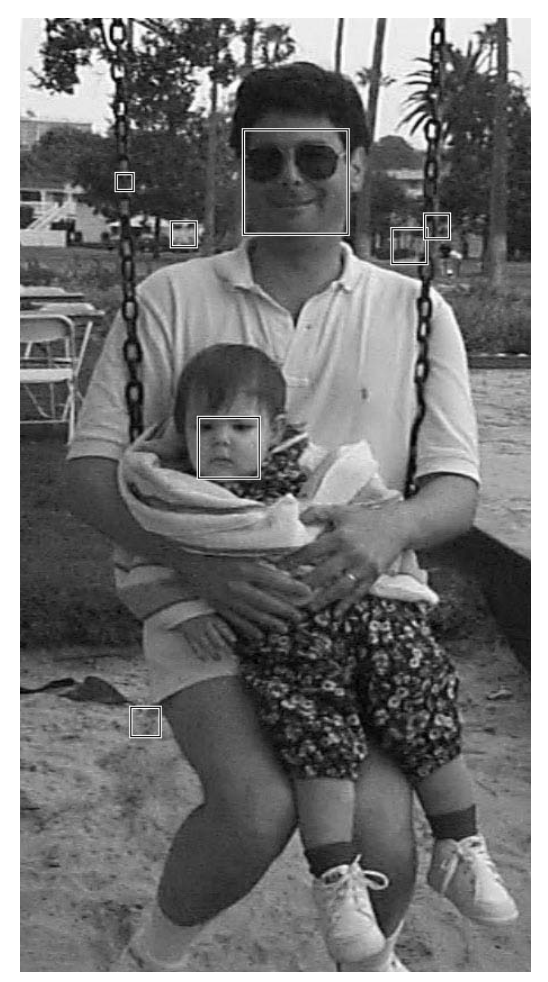

Figure 9: Face detection with the MRC - Example 5

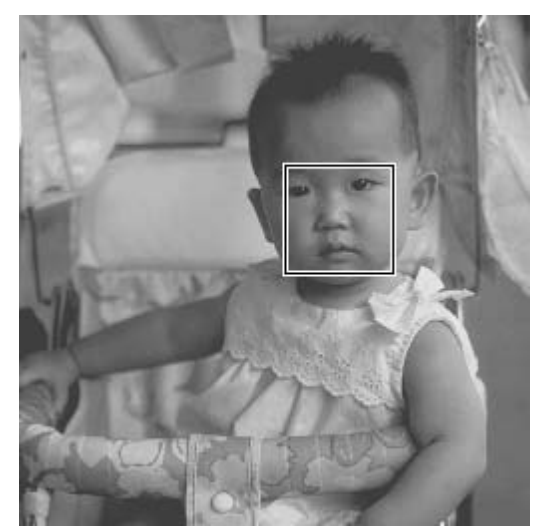

Figure 10: Face detection with the MRC - Example 6 
a face. Generally speaking, the algorithm performs very well in terms of detection rate, false alarm rate, and most important of all, computational complexity.

\section{Conclusion}

In this paper we presented a new classifier for target detection, which discriminates between Target and Clutter classes. The proposed classifier exploits the fact that the probability of a given input to belong to the Target class is much lower, as compared to its probability to belong to the Clutter class. This assumption, which is valid in many pattern detection applications, is exploited in designing an optimal classifier that detects Target signals as fast as possible. Moreover, exact classification is possible when the Target and the Clutter classes are convexly separable. The Fisher Linear Discriminant (FLD) is a special case of the proposed framework when the Target and Clutter probabilities are equal. In addition, the proposed scheme overcomes the instabilities arising in the FLD in cases where the mean of the two classes are close to each other. An improvement of the proposed technique is possible by rejecting Target patterns instead of Clutter patterns in advanced stages, when the probability of Clutter is not larger anymore.

The performance of the MRC is demonstrated in the face detection problem. The obtained face detection algorithm is shown to be both computationally very efficient and accurate.

Further details on the theory of the MRC and its application to face detection can be found in $[14,15]$. 


\section{Acknowledgments}

The authors would like to thank Olliveti Research Laboratory (ORL) for making their database available through the net with no restrictions. Thanks are also in order to Dr. HonjZiang Zhang from Microsoft Research Center in China, for his helpful suggestions and fruitful discussions.

\section{$7 \quad$ References}

[1] R. O. Duda and P. E. Hart, Pattern Classification And Scene Analysis. Wiley-Interscience Publication, 1973. 1st Edition.

[2] V. N. Vapnik, The Nature of Statistical Learning Theory. Springer-Verlag, 1995. 1st Edition.

[3] S. Baker and S. Nayar, "Pattern rejection," in Proceedings 1996 IEEE Computer Society Conference on Computer Vision and Pattern Recognition - San Francisco, CA, USA, 18-20 June, 1996.

[4] G. H. Golub and C. F. V. Loan, Matrix Computations. Johns Hopkins University Press, 1996. 3rd Edition.

[5] J. W. Demmel, Applied Numerical Linear Algebra. SIAM - Society for Industrial and Applied Mathematics, 1997. 1st Edition.

[6] E. Osuna, R. Freund, and F. Girosi, "Training support vector machines: An application to face detection," in IEEE Conference on Computer Vision and Pattern Recognition, PuertoRico, 1997.

[7] H. Rowley, S. Baluja, and T. Kanade, "Neural network-based face detection," IEEE Trans. Pattern Analysis and Machine Intelligence, vol. 20, pp. 23-38, 1998. 
[8] H. Rowley, S. Baluja, and T. Kanade, "Rotation invariant neural network-based face detection," in Calnegie Mellon University Internal Report - CMU-CS-97-201, 1997.

[9] P. Juell and R. March, "A hirarchial neural network for human face detection," Pattern Recognition, vol. 29, pp. 781-787, 1996.

[10] K. Sung and T. Poggio, "Example-based learning for view-based human face detection," IEEE Trans. Pattern Analysis and Machine Intelligence, vol. 20, pp. 39-51, 1998.

[11] A. Mirhosseini and H. Yan, "Symmetry detection using attributed graph matching for a rotation-invariant face recognition system," in Conference on Digital Image Computing: Techniques and Applications - Australia, 1995.

[12] A. Rajagopalan, K. Kumar, J. Karlekar, R. Manivasakan, and M. Patil, "Finding faces in photographs," in International Conference of Computer Vision (ICCV) 97, Delhi, India, 1997.

[13] A. Tankus, H. Yeshurun, and N. Intrator, "Face detection by direct convexity estimation," in Tel-Aviv University Technical Report, 1998.

[14] M. Elad, Y. Hel-Or, and R. Keshet, "Approximated invariant method (AIM): A rejection based classifier," in Hewlett-Packard Technical Report - HPL-98-160, 1998.

[15] M. Elad, R. Keshet, and Y. Hel-Or, "Frontal and vertical face detection in images using the approximated invariant method (aim)," in Hewlett-Packard Technical Report - HPL-99-5, 1999. 